\title{
HIGHLIGHTS
}

SYNAPTIC PLASTICITY

\section{One eye seeing}

We know a great deal about how specific patterns of activity, at specific synapses, can lead to lasting increases or decreases in synaptic strength. We also know a fair amount about how sensory experience - or training can lead to changes in behaviour or in neural responsiveness. But linking the two has proved difficult. Now Heynen et al., writing in Nature Neuroscience, provide compelling evidence for a connection between longterm depression (LTD) at cortical synapses and functional plasticity in the developing visual cortex.

Forty years ago, Wiesel and Hubel discovered that closing one of a kitten's eyes during a 'critical period' could cause remarkable changes in the visual cortex. In areas of cortex that normally responded equally to both eyes, the input from the open eye became dominant as the closed eye lost its influence over the cortical neurons. Later work established that as little as $24 \mathrm{~h}$ of monocular deprivation could have this effect and, crucially, that it depended on the residual, uncorrelated activity in the retina of the closed eye: blocking this activity with tetrodotoxin (TTX) prevented the shift in dominance.

Heynen and colleagues set out to test whether this cortical plasticity is caused by long-term depression at synapses in the visual cortex. LTD is a lasting reduction in synaptic efficacy that, in this case, results from a particular pattern of stimulation of the input nucleus to the visual cortex, the lateral geniculate nucleus. It has a dis- tinctive molecular fingerprint: in neurons that have undergone LTD, AMPA ( $\alpha$-amino-3-hydroxy-5methyl-4-isoxazole propionic acid) receptors show a specific pattern of phosphorylation and dephosphorylation that is associated with internalization of the receptors and decreased synaptic transmission.

The authors found that monocular deprivation reproduced this pattern of effects in the visual cortex. Ocular dominance plasticity, and the molecular effects produced, also mimicked LTD in that it was blocked by treatment of the retina with TTX or by NMDA ( $N$-methyl-D-aspartate) receptor blockade. Another indication that LTD and the changes in ocular dominance share fundamental mechanisms came from the finding that monocular deprivation could occlude the later induction of LTD in brain slices.
Although this work supports the idea that molecular changes resembling LTD are responsible for the initial effects of monocular deprivation — they appear within $6 \mathrm{~h}$ - the molecular changes disappeared after two days. In the longer term, monocular deprivation causes changes in axonal arbours, so it will be fascinating to see whether LTD can act as a trigger for such anatomical plasticity. The mechanisms involved might also point towards a better understanding of how the critical period is controlled during development.

Rachel Jones

\section{(3)) References and links}

ORIGINAL RESEARCH PAPER Heynen, A. J. et al. Molecular mechanism for loss of visual cortical responsiveness following brief monocular deprivation. Nature Neurosci. 6, 854-862 (2003) FURTHER READING McAllister, A. K. \& Usrey, W. M. Depressed from deprivation? Look to the molecules... Nature Neurosci. 6, 787-788 (2003)

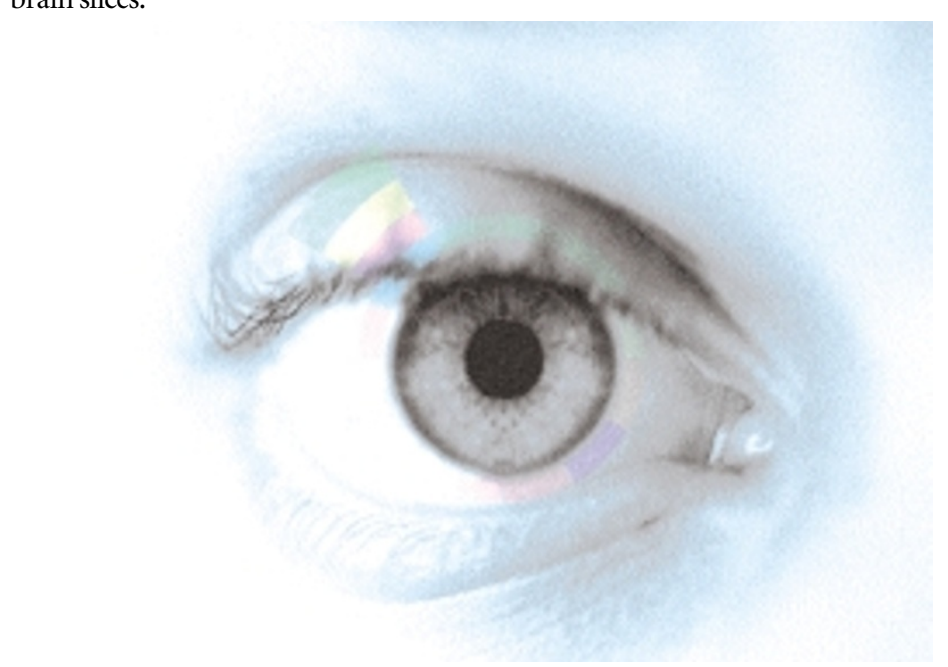

